Optical Spectroscopy Approach for the Predictive Assessment of Kidney

Functional Recovery Following Ischemic Injury

R. N. Raman, C. D. Pivetti, A. M. Rubenchik, D. L. Matthews, C. Troppmann, S. G. Demos

February 19, 2010

Optical Biopsy VIII at Photonics West 2010 San Francisco, CA, United States January 23, 2010 through January 28, 2010 
This document was prepared as an account of work sponsored by an agency of the United States government. Neither the United States government nor Lawrence Livermore National Security, LLC, nor any of their employees makes any warranty, expressed or implied, or assumes any legal liability or responsibility for the accuracy, completeness, or usefulness of any information, apparatus, product, or process disclosed, or represents that its use would not infringe privately owned rights. Reference herein to any specific commercial product, process, or service by trade name, trademark, manufacturer, or otherwise does not necessarily constitute or imply its endorsement, recommendation, or favoring by the United States government or Lawrence Livermore National Security, LLC. The views and opinions of authors expressed herein do not necessarily state or reflect those of the United States government or Lawrence Livermore National Security, LLC, and shall not be used for advertising or product endorsement purposes. 


\title{
Optical spectroscopy approach for the predictive assessment of kidney functional recovery following ischemic injury
}

\author{
Rajesh N. Raman ${ }^{1}$, Christopher D. Pivetti ${ }^{2}$, Alexander M. Rubenchik ${ }^{1}$, Dennis L. Matthews ${ }^{3}$, \\ Christoph Troppmann ${ }^{2}$, and Stavros G. Demos ${ }^{1}$ \\ ${ }^{1}$ Lawrence Livermore National Laboratory, 7000 East Ave., Livermore, CA 94551 \\ ${ }^{2}$ University of California, Davis Medical Center, Dept of Surgery, Cypress Bldg, 2221 Stockton \\ Blvd, Sacramento, CA 95817 \\ ${ }^{3}$ University of California, Davis, NSF Center for Biophotonics, 2700 Stockton Blvd, Ste 1400, \\ Sacramento, CA 95817
}

\begin{abstract}
Tissue that has undergone significant yet unknown amount of ischemic injury is frequently encountered in organ transplantation and trauma clinics. With no reliable real-time method of assessing the degree of injury incurred in tissue, surgeons generally rely on visual observation which is subjective. In this work, we investigate the use of optical spectroscopy methods as a potentially more reliable approach. Previous work by various groups was strongly suggestive that tissue autofluorescence from NADH obtained under UV excitation is sensitive to metabolic response changes. To test and expand upon this concept, we monitored autofluorescence and light scattering intensities of injured vs. uninjured rat kidneys via multimodal imaging under $355 \mathrm{~nm}, 325 \mathrm{~nm}$, and $266 \mathrm{~nm}$ excitation as well as scattering under $500 \mathrm{~nm}$ illumination. $355 \mathrm{~nm}$ excitation was used to probe mainly NADH, a metabolite, while $266 \mathrm{~nm}$ excitation was used to probe mainly tryptophan to correct for non-metabolic signal artifacts. The ratio of autofluorescence intensities derived under these two excitation wavelengths was calculated and its temporal profile was fit to a relaxation model. Time constants were extracted, and longer time constants were associated with kidney dysfunction. Analysis of both the autofluorescence and light scattering images suggests that changes in microstructure tissue morphology, blood absorption spectral characteristics, and $\mathrm{pH}$ contribute to the behavior of the observed signal which may be used to obtain tissue functional information and offer predictive capability.
\end{abstract}

Keywords: kidney, ischemia, reperfusion, spectral imaging, in vivo

\section{INTRODUCTION}

\subsection{Background}

Ischemia is the condition resulting from inadequate blood flow to the tissue. This condition can be the result of stroke, cardiac arrest, or traumatic injury, for example, or routine surgical procedures such as organ transplantation or vascular surgery. Prolonged ischemia can compromise tissue function. At present there is no tool in the clinic or field which can measure the degree of ischemic injury incurred in tissue or predict the return of function at the time of medical intervention. One option available to the surgeon is to excise a sample of the tissue and perform histopathologic analysis to look at morphological changes in the tissue in response to ischemia. However, preparation and analysis typically take days to complete, and such response time is not practical for transplantation or emergency trauma cases. A real-time alternative is microdialysis, a procedure in which the surgeon penetrates the intact tissue with a needle $\sim 2 \mathrm{~mm}$ in diameter in order to sample the extracellular environment for ischemia by-products. Both of these options are invasive and can alter local tissue hemodynamics and metabolism [1]. When dealing with tissue of critical or unknown status, a noninvasive method is preferred. Therefore, the most prevalent method for estimating the degree of ischemic injury in the clinic is based on the visual appearance of the tissue, which is both qualitative and subjective. The repercussions of misjudging tissue ability to function in the transplant clinic can be severe. For example, transplanting dysfunctional tissue can increase the morbidity and mortality of the patient and drive up medical cost when the patient falls ill and needs to return to the clinic for additional surgery. Also, discarding tissue based on its appearance when in reality it is functional increases the shortfall of the current organ donor pool relative to demand. In addition, proper therapeutic 
decisions can be made with a more accurate and timely assessment of tissue functional status, as in whether to resuscitate injured tissue for trauma or peripheral vascular disease patients or whether tissue resuscitation would be futile and risk complications threatening patient survival.

We hypothesize that optical spectroscopy methods, namely multimodal spectral imaging, can provide information allowing for noninvasive, noncontact, real-time monitoring of tissue recovery following ischemic injury. This hypothesis is based on early work by Chance et al. [2-5] correlating NADH (reduced nicotinamide adenine dinucleotide) fluorescence intensity to cell metabolic state. Additional research has shown the importance of monitoring multiple parameters during ischemia and reperfusion in order to characterize tissue response [6]. We expand upon this earlier work by monitoring autofluorescence (AF) under various UV excitation wavelengths as well as light scattering (SC) during ischemia and reperfusion in the kidney, the organ with the highest discard rate in cadaveric donor transplantation [7]. We pursue a noncontact method in order to avoid inducing local ischemia as well as altering the scattering properties of the tissue during our measurement, which can occur when contacting the tissue with a fiber probe and which can introduce signal artifacts [8]. In this work, during the course of ischemia and reperfusion in the kidney, we measure the $\mathrm{AF}$ and SC signals under $355 \mathrm{~nm}, 325 \mathrm{~nm}$, and $266 \mathrm{~nm}$ excitations, as well as green light SC (470-530 nm)

The specific choice for the $355 \mathrm{~nm}$ and $266 \mathrm{~nm}$ excitation wavelengths was based on the commercial availability of these sources in a compact form suitable for eventual transport to and from the clinic. $325 \mathrm{~nm}$ excitation was chosen since it is an isosbestic wavelength with respect to hemoglobin oxygenation ( $355 \mathrm{~nm}$ is not isosbestic), yet still excites NADH.

\section{METHODS}

\subsection{Kidney ischemia model and methods}

All animal procedures were approved by the University of California, Davis, Animal Use and Care Administrative Advisory Committee (AUCAAC). Adult male Spontaneously Hypertensive Rats (SHR) aged 16-17 weeks and weighing 350-400 g were allowed free access to standard pellet chow and tap water. Rats were placed under general anesthesia using $2 \%$ isoflurane delivered with $1.0 \mathrm{~L}$ of air per minute. Depth of anesthesia was monitored by toe pinch tests given at 15 minute intervals during the procedure. Exhaled gases were disposed of appropriately using a filter and scavenging system. Following anesthesia induction, the animal's chest and abdomen were shaved and prepared with alternating rounds of iodine scrub and $70 \%$ ethanol. Next, a midline laparotomy was performed, the small bowel exteriorized, and the left renal pedicle exposed. A non-fluorescent black cloth was then placed over the surrounding tissue to prevent nonkidney tissue autofluorescence. In order to keep the abdominal cavity moist, the intestine was wrapped in saline-soaked gauze, and saline was dripped on the kidneys at 5 minute intervals starting at $\mathrm{t}=10 \mathrm{~min}$. During the monitoring period, the rat was kept warm on a heating pad.

A bulldog clamp was used to occlude arterial and venous flow resulting in unilateral ischemic injury to the left kidney, henceforth referred to as the injured kidney. The right kidney was not clamped and remained undisturbed (normal kidney). Ischemia was induced for either $20(\mathrm{n}=9$ rats $)$ or $150(\mathrm{n}=10)$ minutes. At the end of the injury phase, the clamp was released and the kidneys were allowed to reperfuse. Following reperfusion (at least $45 \mathrm{~min}$ following a $20 \mathrm{~min}$ injury, and at least $60 \mathrm{~min}$ following a $150 \mathrm{~min}$ injury), the normal kidney was removed (contralateral nephrectomy). Post-operatively, rats were given $0.3 \mathrm{cc}$ Buprenex pain medication. Rat weight, physical appearance, and survival were monitored for 30 days following ischemia, or until rat lost more than $20 \%$ of pre-operative body weight (at which time it was sacrificed by exsanguination), or until death. Death by renal failure was determined by timing of death, kidney inspection following death, and subsequent histological analysis.

\subsection{Optical spectroscopy methods}

A schematic layout of the experimental arrangement is shown in Fig. 2. Three laser sources provided UV excitation at $355 \mathrm{~nm}, 266 \mathrm{~nm}$, and $325 \mathrm{~nm}$ with average fluences of $0.2 \mathrm{~mJ} / \mathrm{cm}^{2}, 0.2 \mathrm{~mJ} / \mathrm{cm}^{2}$, and $2 \mathrm{~mJ} / \mathrm{cm}^{2}$, respectively. In addition, white light filtered at $500 \pm 30 \mathrm{~nm}$ provided green light illumination. Illumination output was switched electronically using computer-controlled shutters (Fig. 2). Illumination was coupled into a fiber bundle (Ocean Optics, Dunedin, FL) which delivered the excitation light/green light to the tissue. The fiber output, located $45 \mathrm{~cm}$ from the tissue, permitted full illumination of both kidneys. The autofluorescence (AF) (as well as the green light scattering (SC)) images were recorded through a 420-640 nm band-pass filter positioned in front of a liquid nitrogen-cooled CCD camera. This choice of filter was made in order to capture the emission from NADH (centered at $\sim 460 \mathrm{~nm}$ ) as well as to acquire as much of this signal as possible at the longer wavelengths so as to increase signal collection and reduce acquisition time. A quartz 


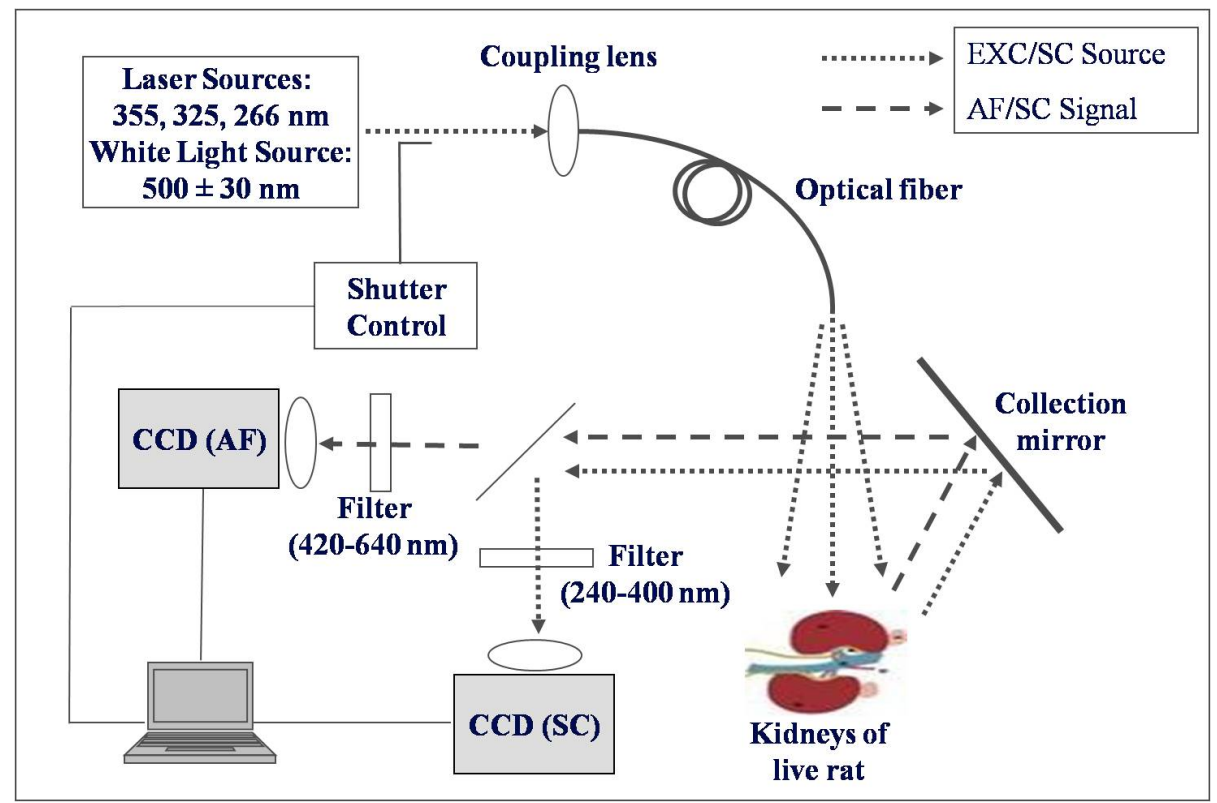

Figure 1. Schematic of experimental setup for multi-modal spectral imaging. EXC=excitation, $A F=$ autofluorescence, $S C=$ scattering.

slide was placed at $45^{\circ}$ to reflect some of the signal to a second CCD in order to monitor the scattered excitation light. A 240-400 $\mathrm{nm}$ band pass filter rejected the autofluorescence portion of this signal. A baseline image was acquired for each illumination source on each camera before the clamp was applied $(t=0)$, then imaging continued immediately upon clamping. Image exposure time was 4 seconds, taken at 15 second intervals over the course of the injury and reperfusion phases.

Each image was then normalized to the beam spatial profile as recorded on a homogeneous fluorescent piece of paper at the start of the experiment as well as normalized to laser power as recorded by a small fluorescent piece of plastic placed alongside the kidneys. Average intensities over as large as possible an area $\left(\sim 0.8 \mathrm{~cm}^{2}\right)$ were recorded for each kidney surface under each illumination source. The region of the image exhibiting specular reflection of the incident light was avoided during analysis since photons scattered from this region do not interact with and therefore do not carry any information on the tissue beneath.

\section{RESULTS AND DISCUSSION}

\subsection{Optical response of injured and normal kidneys}

Figure 2 shows typical results of the effect of 150 min of ischemia and 90 min reperfusion on the AF and SC signals from the injured kidney of one rat. Each trace was normalized to its value before clamping. The green light scattering signal is shown as green triangles and contains periodic spikes which correlate with the application of saline every 5 $\mathrm{min}$. The $500 \mathrm{~nm}$ SC signal changes only a small amount $(\sim 10 \%)$ in the normal kidney (not shown), suggesting that changes in blood volume in this kidney is relatively small. Thus, AF under $355 \mathrm{~nm}$ and $325 \mathrm{~nm}$ excitations in the normal kidney are relatively free from modulation from absorption by blood and likely represent changes in NADH concentration, which slightly increase over the course of injury. This behavior may be due to the increased load the normal kidney experiences during the ischemia phase. In addition, the signal also appears to be sensitive to adjustments we make in the amount of anesthesia delivered as a result of (and to counter) observed changes in the animal's breathing rate during these long experiments. Both of these effects involve a change in [NADH] and tissue metabolism.

The green light scattering signal of the injured kidney in Fig. 2 decreases significantly during injury, suggesting a large increase in local blood volume during the first $20 \mathrm{~min}$. While total blood volume cannot change due to the clamp placement, the blood volume in the capillaries (which are located primarily in the outermost region of the kidney) may increase as a result of capillary response to ischemia which is to dilate. Blood vessels (including microvessels) dilate in 


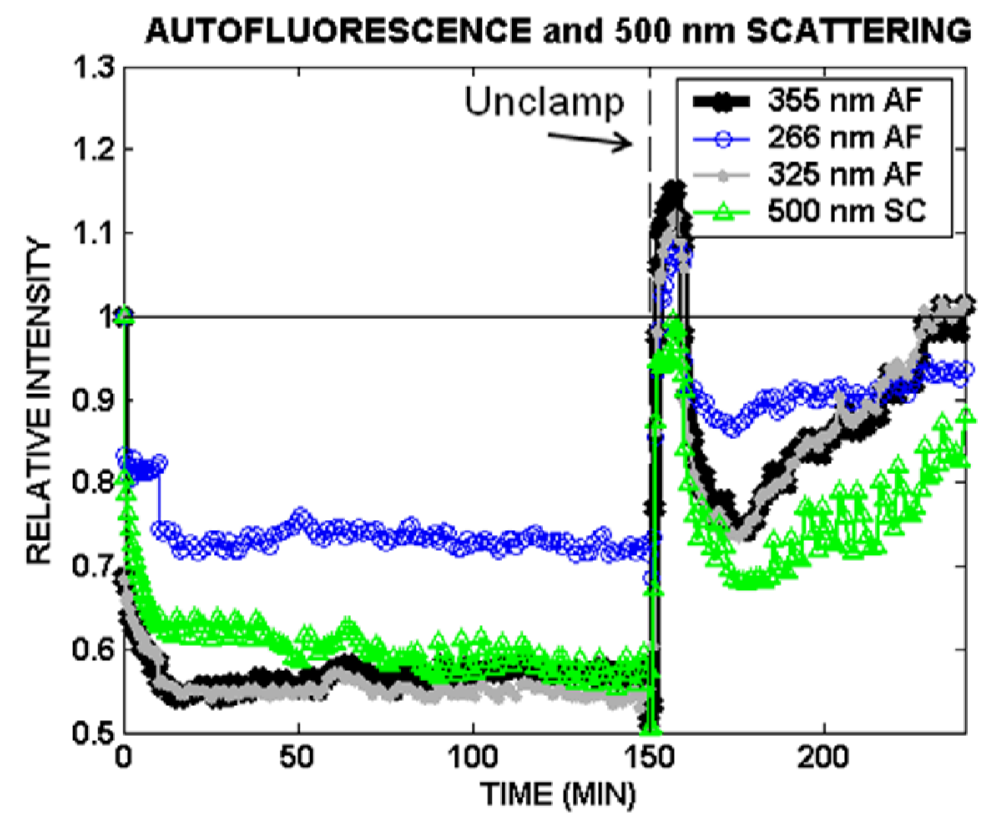

Figure 2. Typical results showing the autofluorescence and green light scattering signals by the injured kidney in one rat undergoing $150 \mathrm{~min}$ ischemia followed by $90 \mathrm{~min}$ reperfusion. The illumination wavelength giving rise to each signal is specified in the legend.

order to increase blood flow (and therefore oxygen delivery) under conditions of oxygen deficiency. This dilation would cause the blood to collect in the newly-created space. Late into injury the green light scattering continues to decrease albeit more slowly. This signal decrease correlates with the excessive darkening of the kidney observed visually towards the end of long injuries. Since blood maximally deoxygenates within $\sim 15 \mathrm{~min}$, this persisting darkening of the kidney may be related to coagulation of the blood. We have observed evidence of coagulation in a few cases where regions of the kidney do not reperfuse satisfactorily after such a long duration of ischemia, suggesting that perhaps blood optical properties are modified after extreme ischemic injuries. Upon unclamping, there appears to be a decrease in the blood volume in the surface capillaries as these microvessels respond to the return of oxygenated blood and constrict back towards a much smaller diameter, followed by a subsequent phase of dilation and final constriction phase as these vessels finds their equilibrium homeostatic diameter. It is interesting to note that the $500 \mathrm{~nm} \mathrm{SC}$ signal essentially returns back to its pre-injury value, indicating that tissue color at the end of the reperfusion phase carries no history of the actual injury process and that therefore visual observation can be misleading.

These changes in blood volume appear to influence the AF under $355 \mathrm{~nm}$ and $325 \mathrm{~nm}$ excitations, shown as black crosses and gray stars, respectively, during injury as well as during parts of reperfusion. However, the magnitude of the increase is higher and the rate is faster for the NADH signal versus the green light scattering signal, suggesting that a factor other than change in blood volume is contributing to the observed behavior. The AF under $266 \mathrm{~nm}$ excitation also appears to be somewhat sensitive to injury and reperfusion but much less so than that under $355 \mathrm{~nm}$ excitation. This slight sensitivity may be due to modulation by blood as well as a minor contribution from NADH fluorescence. NADH has an absorption peak near $266 \mathrm{~nm}$ corresponding to a fluorescence peak $440 \mathrm{~nm}$ [12-13]. However, because of tryptophan's relatively high fluorescence efficiency, the emission under excitation near $266 \mathrm{~nm}$ is dominated by tryptophan [14]. A direct comparison of the AF under $355 \mathrm{~nm}$ against that under $325 \mathrm{~nm}$ shows only minor differences. These small changes during the injury and reperfusion phases suggest that changes in blood oxygenation state do not significantly affect the NADH signal.

Figure 3 shows the temporal response of the excitation light scattering signals to ischemia and reperfusion in the same rat described in Fig. 2. Firstly, one can see the dramatic transient effect of saline for the cases of the scattered $355 \mathrm{~nm}$ and $325 \mathrm{~nm}$ wavelengths. It has previously been suggested that the scattered excitation light can provide a reference 


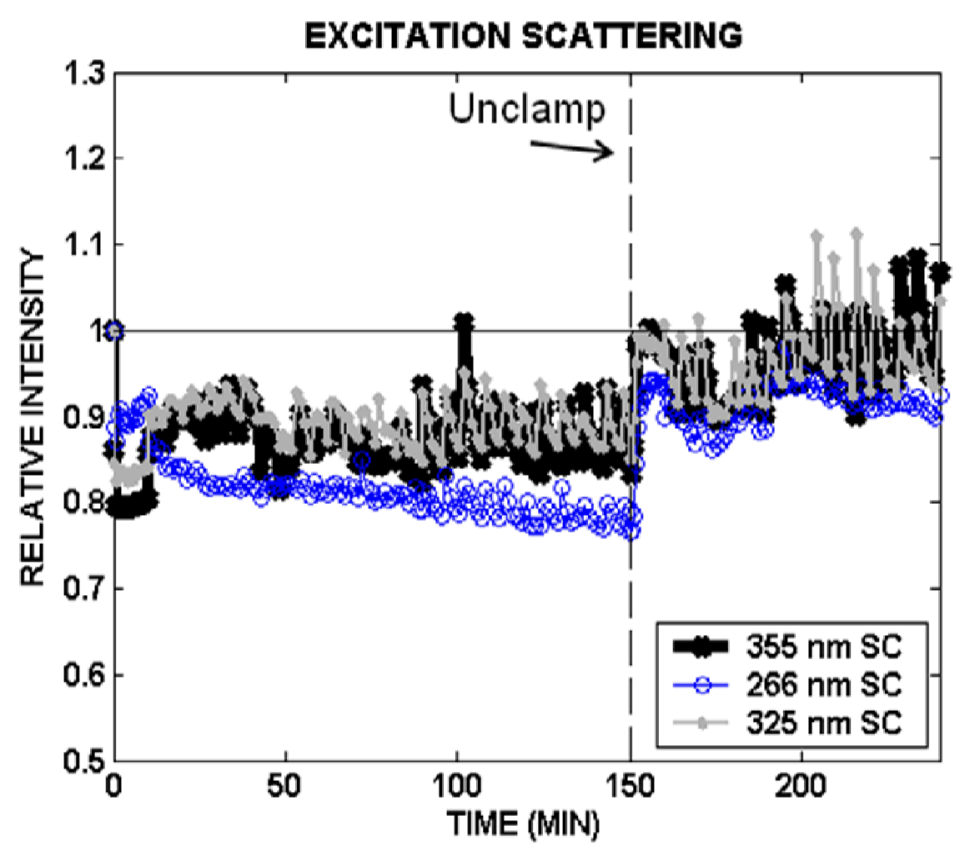

Figure 3. Light scattering of the UV excitation sources by the injured kidney.

signal which can account for changes in blood volume [15]. While perhaps showing promise in a method in which the measurement instrument is in contact with the tissue surface, in a noncontact method such as in imaging or in a noncontact fiber optic probe method [16], the scattered excitation light serves as a poor reference signal and introduces periodic artifacts when the tissue is hydrated. This artifact is particularly a concern in the clinic in which tissue must be continually hydrated during long procedures where the tissue is exposed to the air for extended periods. In addition, two discontinuities are observed. The second one relates to tissue hydration and occurs after $\mathrm{t}=10 \mathrm{~min}$ in most of the recorded signals when the administering of saline begins. The first one occurs immediately after $\mathrm{t}=0$ and is likely a movement artifact as the weight of the clamp moves the kidney and changes the illumination/collection geometry slightly.

Secondly, the profiles of the scattered excitation light from the injured kidney resembles, though much less in magnitude, the green light scattering profile. This observation suggests that there is a small degree of modulation of the excitation light by blood absorption. Also, it is known that scattering centers in the tissue change size during ischemia (e.g. microvessels, mitochondria) and may be contributing to the scattering signal. Evidence of wavelength-dependent scattering can be observed whenever the behavior of the $355 \mathrm{~nm} \mathrm{SC}$ signal varies from that of the $266 \mathrm{~nm}$ SC signal. The precise effect of swelling and shrinking of organelles, microvascular, and other components is currently under study using models of tissue scattering.

Finally, one sees that upon comparing the AF under $355 \mathrm{~nm}$ to that under $266 \mathrm{~nm}$, the discontinuities observed in the original NADH signal (Fig. 2) after clamping and after the application of saline, signal artifacts which are not related to metabolism, would be largely removed by division while the characteristic injury- and recovery-related NADH signal features would be maintained. These characteristic features include the two-phase optical recovery following removal of the clamp. This result was expected since emission under $266 \mathrm{~nm}$ (dominated by tryptophan) should be relatively unaffected by a change in metabolic state during ischemia and reperfusion and should therefore provide a reference signal against which to normalize non-metabolic artifacts. Thus, this ratio formed by dividing the emission intensity of the injured kidney under $355 \mathrm{~nm}$ excitation to that under $266 \mathrm{~nm}$ excitation (henceforth referred to as the signal ratio) was calculated for all of the rats in this study for both the $20 \mathrm{~min}$ and 150 min injury cases.

\subsection{Analysis of the signal ratio}

We are primarily interested in the tissue optical properties during the reperfusion phase, since this period represents the phase of medical intervention. We observed that for 150 min injury, the two-phase optical recovery of the signal ratio 
Table I. Comparison of results from fits of the signal ratio to the double exponential model, followed by corresponding survival outcome for two rats each subjected to different durations of ischemia specified on the left-hand side.

\begin{tabular}{|c|c|c|c|c|}
\hline Ischemia time & $\boldsymbol{\Delta} \boldsymbol{\tau}(\mathbf{m i n})$ & $\boldsymbol{\tau}_{\mathbf{N}}(\mathbf{m i n})$ & $\boldsymbol{\tau}_{\mathbf{E}}(\mathbf{m i n})$ & Day of Death \\
\hline 20 min & 1.2 & 1.8 & 5.5 & N/A \\
\hline & & & & (Survived) \\
\hline
\end{tabular}

consistently exhibited features distinct from that for the case of 20 min injury. Namely, the time for the signal ratio to return to its first peak upon unclamping as well as the time interval between the arrival of this first peak and reaching its final value tended to be longer for the case of 150 min injury. Therefore, we compared the dynamics of the signal ratio during reperfusion for each rat according to a kinetic model described in detail elsewhere [16]. In brief, the signal ratio was modeled as the product of two components, each represented by an exponential function. The first component represents the contribution to the signal ratio from [NADH] and is described by two fitting parameters: a delay time, $\Delta \tau$, and a relaxation time, $\tau_{\mathrm{N}}$. The delay time represents a delay in the oxidation of $\mathrm{NADH}$, either as a result of delayed oxygenated blood flow to the tissue upon unclamping or the delay in the re-establishment of the electron transport chain whose integrity may have been severely compromised following severe injury. The relaxation time $\tau_{\mathrm{N}}$ represents the time for the amount of NADH to decrease to 1/e of its initial value upon unclamping. The second component represents the influence of the tissue environment on the signal ratio, which itself may be the composite of the environmental factors described in section 3.1. This second component is described by a relaxation time, $\tau_{\mathrm{E}}$, which represents the amount of time for the contribution from modulation to increase to $1 / \mathrm{e}$ of its final value. Thus, fits of the data to this model yield three time constants as fitting parameters.

The results of this analysis and survival outcome for two rats, one undergoing a 20 min injury while the other undergoing a 150 min injury, are summarized in Fig. 4. The signal ratio of the rat undergoing prolonged injury was characterized by longer time constants. Visual comparison of the injured kidneys of these two rats in vivo revealed strikingly similar color and intensity within $10 \mathrm{~min}$ of clamp release, and furthermore, each injured kidney resembled the corresponding normal kidney. This resemblance is despite the fact that the $150 \mathrm{~min}$ injury proved to be a fatal injury, as this rat died of kidney failure 5 days after the injury (Table I). Preliminary results indicate that, as found previously [16], prolonged injury on average tend to yield longer time constants. In addition, the results of the survival study show preliminarily that on average, rats exhibiting longer time constants (obtained within 90 min of reperfusion) are more likely to die of kidney failure, which in our experiments generally occurred between 3-7 days following the experiment, while those which undergo 20 min injury are more likely to survive.

\section{CONCLUSIONS}

In this work, multispectral imaging during kidney ischemic injury and reperfusion provided information relating to tissue function. Namely, analysis of the dynamics of the signal ratio formed by dividing the autofluorescence under $355 \mathrm{~nm}$ to that under $266 \mathrm{~nm}$ has shown that kidneys subjected to prolonged injury respond during reperfusion with slower autofluorescence dynamics. This information can be used to predict kidney failure when visual observation cannot, and it can be used to do so long before death due to kidney failure. In addition, multispectral imaging has provided insight into various physiological events which may be occurring during ischemia and reperfusion that are responsible for the observed behavior of the signal ratio. Results indicate that absorption of the emission by blood is occurring, likely due to vascular response to injury and reperfusion. However, late into reperfusion, other factors appear to be modulating the signal which may be affecting fluorophore quantum efficiency. Blood oxygenation state has minimal influence on the dynamics of the signal ratio throughout the injury and recovery processes. Further modeling may confirm the origin of the wavelength dependence of the scattered signals as arising from scattering centers which change in size in response to ischemic conditions. 


\section{ACKNOWLEDGEMENTS}

This work was performed under the auspices of the U.S. Department of Energy by Lawrence Livermore National Laboratory under Contract DE-AC52-07NA27344. This work has been supported in part by the Center for Biophotonics, an NSF Science and Technology Center, managed by the University of California, Davis, under Cooperative Agreement number PHY0120999. This work was also supported in part by a research grant from the Transplant Hope Endowment, University of California, Davis, Medical Center, Sacramento.

\section{REFERENCES}

[1] Sommer T. and Larsen, J. F., "Detection of intestinal ischemia using a microdialysis technique in an animal model," World J. Surg. 27, 416-420 (2003).

[2] Chance, B., "Spectrophotometry of intracellular respiratory pigments," Science 120, 767-775 (1954).

[3] Chance, B., Cohen, P., Jobsis, F., and Schoener, B., "Intracellular oxidation-reduction states in vivo," Science 137, 499-508 (1962).

[4] Chance, B., Williamson, J. R., Jamieson, D., and Schoener, B., "Properties and kinetics of reduced pyridine nucleotide fluorescence of the isolated and in vivo rat heart" Biochem. Z. 341, 357-377 (1965).

[5] Kobayashi, S., Nishiki, K., Kaede, K., and Ogata, E., "Microfluorometry of oxidation-reduction state of the rat kidney in situ," J. Appl. Physiol. 31, 693-696 (1971).

[6] Mayevsky, A., Nakache, R., Luger-Hamer, M., Amran, D., and Sonn, J., "Assessment of transplanted kidney vitality by a multiparametric monitoring system," Transplant. Proc. 33, 2933-2934 (2001).

[7] Ojo, A. O., Heinrichs, D., Emond, J. C., McGowan, J. J., Guidinger, M. K., Delmonico, F. L., and Metzger, R. A., "Organ donation and utilization in the USA," Am. J. Transplant. 4, 27-37 (2004).

[8] Reif, R., Arnorosino, M. S., Calabro, K. W., A'Amar, O., Singh, S. K., and Bigio, I. J., "Analysis of changes in reflectance measurements on biological tissues subjected to different probe pressures," J. Biomed. Opt. 13, 07424LR (2008).

[9] Stratford, M. R. L., Parkins, C. S., Everett, S. A., Dennis, M. F., Stubbs, M., and Hill, S. A., "Analysis of the acidic microenvironment in murine tumors by high-performance ion chromatography," J. Chromatogr. A 706, 459-462 (1995).

[10] Eklund, T., Wahlberg, G., Ungerstedt, U., and Hillered, L., "Interstitial lactate, inosine, and hypoxanthine in rat kidney during normothermic ischaemia and recirculation," Acta Physiol. Scand. 143, 279-286 (1991).

[11] Mehrabi, A., Golling, M., Busch, C., Hashemi, B., Ahmadi, R., Volkl, A., Gebhard, M. M., Klar, E., and Kraus, T., "Experimental monitoring of hepatic metabolism by microdialysis glucose, lactate, and glutamate during surgical preparation of the liver hilus," J. Surg. Res. 105, 128-135 (2002).

[12] Alfano, R. R. and Yang, Y., "Stokes shift emission of human tissue and key biomolecules," IEEE J. Quantum Elect. 9, 148-153 (2003).

[13] Richards-Kortum, R. and Sevick-Muraca, E., "Quantitative optical spectroscopy for tissue diagnosis," Annu Rev Phys. Chem. 47, 555-606 (1996).

[14]Fitzgerald, J. T., Michalopoulou, A. P., Pivetti, C. D., Raman, R. N., Troppmann, C., and Demos, S. G., "Realtime assessment of in vivo renal ischemia using laser autofluorescence imaging," J. Biomed. Opt. 10, 044018 (2005).

[15] Mayevsky, A. and Chance, B., "Intracellular oxidation-reduction state measured in situ by a multichannel fiberoptic surface fluorometer," Science 217, 537-540 (1982).

[16]Raman, R. N., Pivetti, C. D., Matthews, D. L., Troppmann, C., and Demos, S. G., "A non-contact method and instrumentation to monitor renal ischemia and reperfusion with optical spectroscopy," Opt. Express 17, 894-905 (2009). 